Case Report

\title{
Sigmoid Volvulus in a Neonate with Anorectal Malformation: A Case Report
}

\author{
Wilms $M C^{*}$ and Lienert $M$ \\ Department of General and Abdominal Surgery, Sana Kliniken Düsseldorf, Germany
}

\begin{abstract}
Background: Sigmoid volvulus in the neonate is an extremely rare condition needing emergency treatment. Sporadic cases of neonatal sigmoid volvulus and some in association with Hirschsprung's disease have been documented. Sigmoid volvulus has been described only twice in the literature in association with anorectal malformation. However, newborns with anorectal malformations might be especially at risk for sigmoid volvulus due to increased intraluminal intestinal weight caused by bowel obstruction and its existence might be underreported.
\end{abstract}

Case presentation: This is the case report of a full-term neonate with trisomy 21 who was diagnosed with anorectal malformation upon birth. An abdominal $\mathrm{X}$-ray on his second day of life showed typical radiological findings of sigmoid volvulus as described by the radiologist. However, the findings were interpreted as a dilated rectosigmoid sling typical for anorectal malformation by the surgeon and a transverse loop colostomy was placed. On the fourth postoperative day sigmoid perforation occurred and led to meconium peritonitis and septic shock. An emergency laparotomy was performed and a perforation at the distal sigmoid colon was found.

Conclusion: There is an urgent need to raise awareness as to the existence of sigmoid volvulus in the neonate with anorectal malformations. The distinct radiological findings of the "coffee bean sign", the "northern exposure sign" and an empty rectum allow to distinguish the extremely rare sigmoid volvulus from the common finding of a dilated rectosigmoid in patients with anorectal malformations. Detorsion of sigmoid volvulus is vital to preventing sigmoid perforation.

Keywords: Sigmoid volvulus; Neonate; Anorectal malformation; Sigmoid perforation

\section{Introduction}

Acute sigmoid volvulus is a well-known emergency in the adult population, occurring when an elongated sigmoid colon twists around the axis of a proportionally short mesenteric root. These pathological anatomical alterations are more common in elderly patients with a history of chronic constipation [1]. It is therefore not surprising that sigmoid volvulus in the neonate is a rare condition. In the literature, sigmoid volvulus in the neonate is documented predominantly in association with Hirschsprung's disease [2,3]. The concept that sigmoid volvulus in the neonate can arise from any condition that causes bowel obstruction has been introduced before [4]. However, to our knowledge, only two previous cases have reported a sigmoid volvulus in a neonate with Anorectal Malformation (ARM) $[4,5]$.

The term anorectal malformation comprises a range of congenital abnormalities that affect the anus and rectum and can additionally involve the urogenital tract. The majority of neonates with ARM require surgery within the first $48 \mathrm{~h}$ of life to resolve bowel obstruction,

Citation: Wilms MC, Lienert M. Sigmoid Volvulus in a Neonate with Anorectal Malformation: A Case Report. J Surg Surgic Case Rep. 2021;2(1):1015.

\section{Copyright: (c) 2021 Wilms MC}

Publisher Name: Medtext Publications LLC

Manuscript compiled: Jul $28^{\text {th }}, 2021$

*Corresponding author: Wilms MC, Department of General and Abdominal Surgery, Sana Kliniken Düsseldorf, Gräulinger Straße 120, Düsseldorf 40625, Germany, Tel: +49-211-2800-1; Fax: +49-211-2800-3301; E-mail: miriam.wilms@gmx.de thereby necessitating a decision as to whether a colostomy is needed or whether a primary Posterior Sagittal Anorectoplasty (PSARP) can be performed. Early decision-making requires a thorough clinical evaluation and an ultrasound of the abdomen. An X-ray of the sacral spine is routinely recommended; an abdominal X-ray is recommended if the abdominal findings are suspicious [6].

Detection of sigmoid volvulus in the neonate through clinical findings alone needs a high level of suspicion of this rare differential diagnosis. This is especially true in the presence of ARM, as clinical signs of sigmoid volvulus such as abdominal distension, and failure to pass meconium overlap with possible abdominal findings of ARM [5]. Sigmoid volvulus can be diagnosed, showing the pathognomonic "coffee bean sign", the "northern exposure sign" (apex of the sigmoid volvulus cranial to the transverse colon) and an empty rectum on abdominal X-ray. These radiological signs allow to distinguish sigmoid volvulus from a dilated rectosigmoid sling that can often be found in ARM. Awareness of the occurrence of sigmoid volvulus in association with ARM in the neonate is essential to its proper detection and management.

\section{Case Presentation}

A 7-month-old boy initially presented to our hospital for an ARM follow-up visit. His medical history reported an ARM without fistula that was treated with a transverse loop colostomy on his second day of life. Sigmoid perforation on his fifth day of life led to meconium peritonitis and septic shock with multiorgan failure. Because the occurrence of sigmoid perforation with peritonitis four days after the placement of a diverting colostomy is highly unusual, we performed a thorough review of the medical history.

The diagnosis of trisomy 21 was made through amniocentesis at 22 weeks of gestation and no malformations were detected on prenatal 
ultrasound. The boy was born at $37+0$ weeks of gestation, weighing $2.48 \mathrm{~kg}$. An ARM was visually diagnosed postpartum and there was no clinical suspicion regarding the abdomen. A nasogastric tube and an umbilical venous catheter were placed.

On the second day of life the patient was transferred to a "highvolume center" for anorectal surgery in the newborn.

An abdominal ultrasound suspected a dilated rectosigmoid sling. An abdominal X-ray was taken at 27 hours of life to screen for accompanying malformations (Figure 1), with the radiologist's evaluation being: "... A dilated intestinal sling of up to $3.2 \mathrm{~cm}$ of diameter reminds of the "coffee bean" sign as seen in sigmoid volvulus and is most likely originated in the sigmoid...."

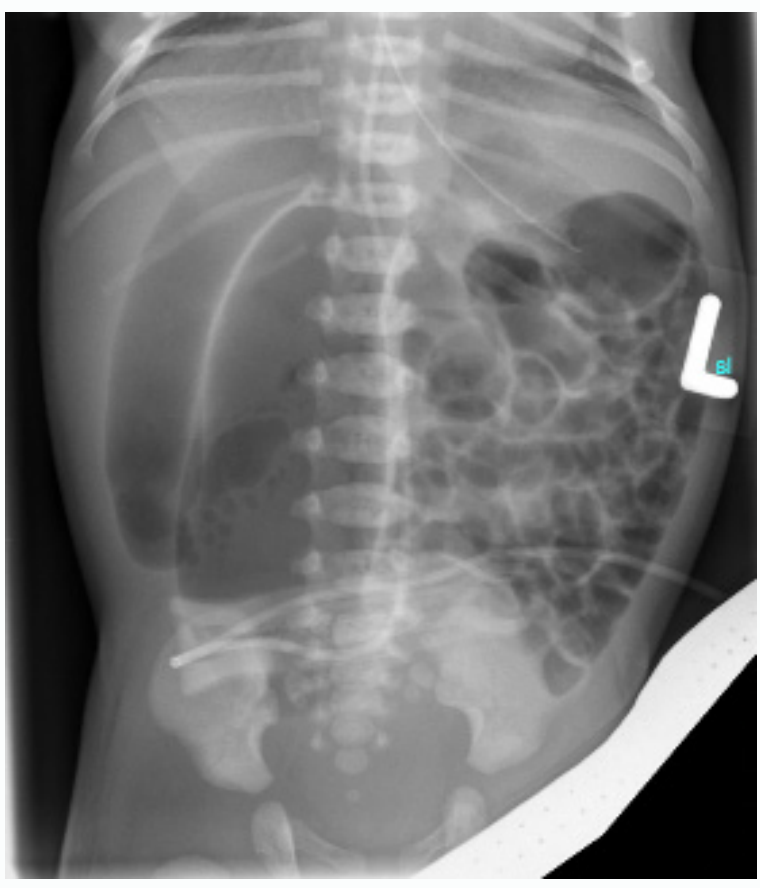

Figure 1: Abdominal X-ray at 27 hours of life showing sigmoid volvulus with pathognomonic "coffee bean sign", "northern exposure sign" (apex of sigmoid volvulus cranial to the transverse colon) and an empty rectum.

At 29 hours of life a transverse colostomy was placed in the right upper quadrant using minilaparotomy through the ostomy side. The intraoperative finding was of a severely distended sigmoid colon in the right upper quadrant, with no signs of gangrene. Despite the intraoperative finding and the preoperative radiological finding of a sigmoid volvulus, no further measures were taken to treat the sigmoid volvulus. An exploration of the entire abdomen could not be performed through minilaparotomy in the right upper quadrant. No further radiological examination was performed during the following four days. Clinical evaluation showed a well-functioning colostomy; however, the enteral alimentation was slow and gastric reflux was noted.

On the fourth postoperative day the general condition of the patient deteriorated and he was centralized and somnolent. Over the course of the night the abdomen distended and in the early morning hours of the fifth postoperative day, a phlegmon of the entire abdomen had formed. The abdominal X-ray showed no intestinal gas, suspicious of meconium peritonitis, and the ultrasound showed large amounts of extraintestinal abdominal fluid. The intraoperative findings of an emergency laparotomy were of a generalized meconium peritonitis; an approximately $5 \mathrm{~mm}$ perforation was detected at the distal sigmoid, but no gangrene of the sigmoid was present. There was no sign of malrotation. A distal colostogram was performed through the side of the perforation, showing no fistula (Figure 2). A thorough lavage was performed and the perforation was sutured. A simultaneous PSARP was undertaken. The patient suffered septic shock with multiorgan failure, and his recovery was prolonged.

At the age of six months the colostomy closure was performed. The preoperative distal colostogram showed a regular, slender and not elongated, sigmoid colon. No recurrence of sigmoid volvulus had occurred at 12 months of life.

\section{Discussion}

Sigmoid volvulus is a life-threatening condition that requires immediate emergency care. Despite sigmoid volvulus being a wellknown entity in the adult population, it is extremely rare in neonates. Ten previous reports of a total of 15 cases of neonatal sigmoid volvulus have been published [2]. In all of the cases the patient was male; six of the patients had Hirschsprung's disease [2], one had ARM without fistula [4], and one had anal stenosis [5]. There was no apparent cause associated with sigmoid volvulus in the remainder of the reported cases.

Clinically the presentation of sigmoid volvulus cannot be distinguished from the presentation of a distended abdomen that is due to ARM. In the case of our patient, an abdominal X-ray was performed and sigmoid volvulus was described by the radiologist. However, intraoperatively the surgeon did not consider sigmoid volvulus despite the dilated sigmoid colon in the right upper quadrant having been noted. The transverse colostomy alone was not a sufficient treatment for the sigmoid volvulus. An irrigation of the relatively long distal loop was not performed.

The unfavorable outcome of this case clearly demonstrates the urgent need to raise awareness as to the existence of sigmoid volvulus in the neonate with ARM, which might be underreported. The distinct radiological findings of the "coffee bean sign", the "northern exposure

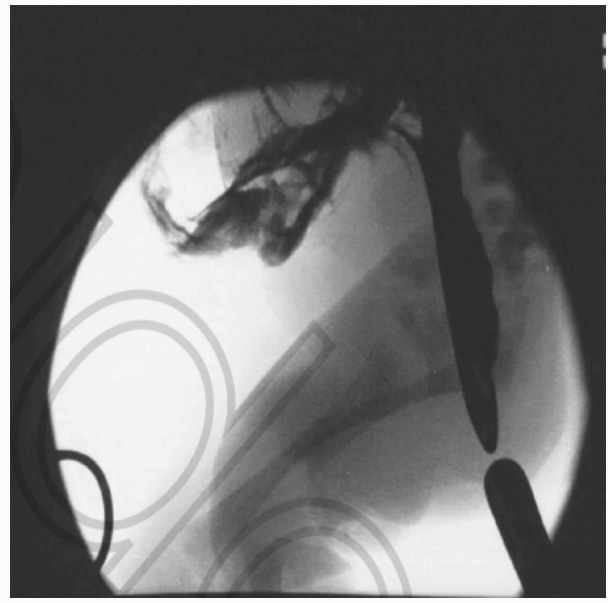

Figure 2: Intraoperative colostogram through the site of the perforation in the distal sigmoid. No fistula is present. 
sign" and an empty rectum (Figure 1) allow to distinguish between the common finding of a dilated rectosigmoid in patients with ARM and the rare finding of sigmoid volvulus.

Furthermore, even in the absence of sigmoid volvulus a loop transverse colostomy should be discouraged as it leaves a relatively long difunctionalized distal colon [6]. A split descending colostomy should be favored.

Previous studies have shown that unlike the adult population in which sigmoid volvulus often causes gangrene, this does not seem to be the case in the neonatal population $[2,3]$. The limited published literature has also shown no recurrence of sigmoid volvulus in the neonate if the underlying cause of bowel obstruction was treated [2], and this observation is supported by our case report. At six months of age, the distal colostogram of our patient did not show an elongation but rather a completely normal, slender sigmoid colon, thus suggesting that the pathogenesis of sigmoid volvulus of the neonate is different to that of sigmoid volvulus in the adult. Increased intraluminal weight of the colon arising from bowel obstruction associated with Hirschsprung's disease or ARM, as examples, seems to be sufficient for sigmoid volvulus to develop in the neonate. This is an important consideration in its management, as it renders sigmoidectomy unnecessary if the associated underlying condition is resolved. De Caluwé et al. [5] could demonstrate a successful detorsion of sigmoid volvulus through contrast enema in a neonate with ARM with perineal fistula. In neonates with sigmoid volvulus and ARM without perineal fistula, we advise for timely open detorsion of the sigmoid volvulus and the placing of a split descending colostomy.

In light of this case report, we would strongly advice against performing a simultaneous PSARP in an emergency laparotomy when generalized meconium peritonitis is present as the risk of septic shock increases.

\section{Conclusion}

Sigmoid volvulus is a rare but important differential diagnosis in neonates with ARM. As clinical evaluation alone is not sufficient to detect sigmoid volvulus, we recommend a routine abdominal X-ray to be taken within the first $48 \mathrm{~h}$ of life in patients with ARM. The distinct radiological findings of sigmoid volvulus should be known to every radiologist, and proper knowledge of this rare differential diagnosis should be established amongst neonatal surgeons. Sigmoid volvulus should be treated by immediate detorsion to avoid sigmoid perforation.

\section{References}

1. Dolejs SC, Guzman MJ, Fajardo AD, Holcomb BK, Robb BW, Waters JA. Contemporary management of sigmoid volvulus. J Gastrointest Surg. 2018;22(8):1404-11.

2. Khalayleh H, Koplewitz B, Kapuller V, Armon Y, Abu-Leil S, Arbell D. Neonatal sigmoid volvulus. J Pediatr Surg. 2016;51(11):1782-5.

3. Venugopal K, Wilcox D, Bruce J. Hirschsprung's disease presenting as sigmoid volvulus in a newborn. Eur J Pediatr Surg. 1997;7(3):172-3.

4. Janik JS, Humphrey R, Nagaraj HS. Sigmoid volvulus in a neonate with imperforate anus. J Pediatr Surg. 1983;18(5):636-8.

5. De Caluwé D, Kelleher J, Corbally MT. Neonatal sigmoid volvulus: a complication of anal stenosis. J Pediatr Surg. 2001;36(7):1079-81.

6. Levitt MA, Peña A. Anorectal malformations. Orphanet J Rare Dis. 2007;2(1):33. 